\title{
Synthesis and Electrochemical Behavior of Some New Pyridazine Derivatives
}

\author{
Essam Abdelghani, Wesam Saber Shehab*, Medhat El-Mobayed, Atef M. \\ Abdel Hamid \\ Chemistry Department, Faculty of Science, Zagazig University, Zagazig, Egypt \\ *E-mail: Dr_wesam123@yahoo.com \\ E-mail: e_abdelghani58@hotmail.com
}

\begin{abstract}
One-pot reaction of $\mathbf{1}$ with ethyl cyanoacetate and/or benzylidenemalono- nitrile afforded pyridazine derivatives $\mathbf{2}$ and $\mathbf{3}$, respectively. Compound $\mathbf{2}$ was subjected to some reactions to produce other new pyridazine derivatives. Also, treatment of $\mathbf{2}$ with hydrazine hydrate in ethanol gave the carbohydrazide 7. Some derivatives of the latter compound have been synthesized. Cyclic voltammograms of compounds 2, 5b, 7 and $\mathbf{8 b}$ using an undivided cell at platinum electrodes are discussed. The antimicrobial activity of some synthesized derivatives has been investigated.
\end{abstract}

Keywords: Pyridazine, Carbohydrazide, Carboxamide, Oxidation, Reduction.

\section{Introduction}

Several functionalized pyridazines exhibit important biological activity such as: antibacterial, antibiotic, antitumor, antiviral and antidiabetes [1]. Pyridazine derivatives could also find application as ligands in supramolecular chemistry and in metallic complexes which exhibit catalytic properties [2-4]. On the basis of these reports and in continuation of our work [5-8]; we synthesized some novel pyridazine derivatives of expected notable chemical, electrochemical and biological activities.

\section{Result and Discussion}

We reported here the synthesis of ethyl 5-cyano-4-methyl-6-oxo-1-phenyl-1,6dihydropyridazine-3-carboxylate

(2) by treatment of ethyl 3-oxo-2- 
(phenyl- hydrazono)butanoate (1a) with ethyl cyanoacetate in the presence of ammonium acetate and acetic acid. Also, 6-acetyl-3-imino-2,5-diphenyl-2,3,4,5tetrahydropyridazine-4-carbonitrile (3) was synthesized by heating of 1(phenylhydrazono)propan-2-one $\mathbf{1 b}$ with benzylidenemalononitrile in pyridine. The structure of compounds $\mathbf{2}$ and $\mathbf{3}$ were established on the basis of their elemental analysis and spectral data. The IR spectrum of compound 2 showed absorption bands at $2230(\mathrm{C} \equiv \mathrm{N}), 1717(\mathrm{C}=\mathrm{O}), 1676(\mathrm{C}=\mathrm{O})$ and $1585(\mathrm{C}=\mathrm{C}) \mathrm{cm}^{-}$ 1

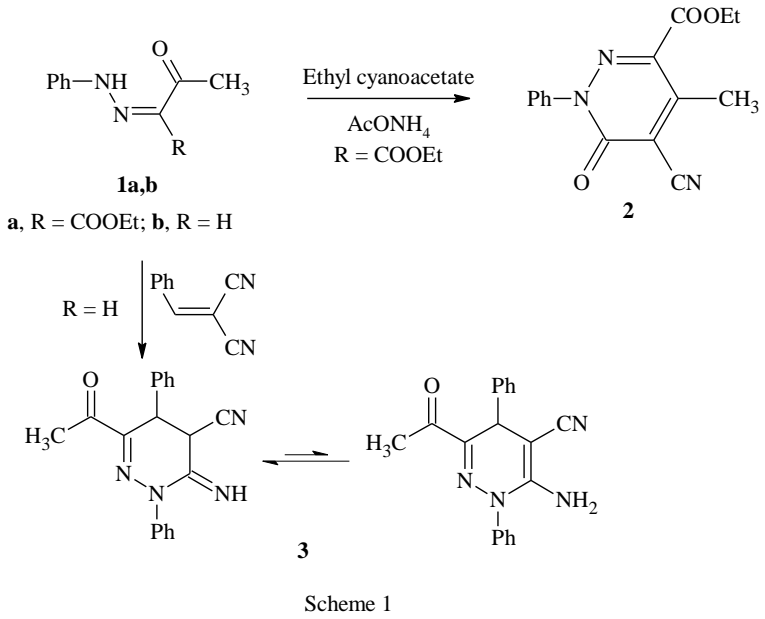

The ${ }^{1} \mathrm{H}$ NMR spectrum of compound 2 showed signals at $\delta 1.28$ and $2.64 \mathrm{ppm}$ characteristic for $2 \mathrm{CH}_{3}$, in addition to signal at $\delta 4.34 \mathrm{ppm}$ as a quartet for $\mathrm{CH}_{2}$ of ethyl group. The IR spectrum of compound $\mathbf{3}$ showed absorption bands at $3407(\mathrm{NH}), 2188(\mathrm{C} \equiv \mathrm{N})$ and $1644 \mathrm{~cm}^{-1}$ for $\mathrm{C}=\mathrm{O}$ (amide). ${ }^{1} \mathrm{H}$ NMR spectrum of 3 showed signals at $\delta 2.32,4.75$ and $5.99 \mathrm{ppm}$ indicate the presence of $\mathrm{CH}_{3}$ and $2 \mathrm{CH}$ groups, in addition to signal at $\delta 7.17 \mathrm{ppm}$ for $\mathrm{NH}$.

Reaction of compound 2 with different reagents namely, sulphur, benzaldehyde, salicylaldehyde, and/or benzylidenemalononitrile, in absolute ethanol and few drops of piperidine afforded ethyl 5-amino-4-oxo-3-phenyl-3,4dihydrothieno[3,4- $d]$ pyridazine-1-carboxylate (4), ethyl 5-cyano-6-oxo-1phenyl-4-[2-phenylethenyl]-1,6-dihydro-pyridazine-3-carboxylate (5a), ethyl

5-cyano-4-[2-(2-hydroxyphenyl)ethenyl]-6-oxo-1-phenyl-1,6dihydropyridazine-3-carboxylate (5b), ethyl 5-amino-6-cyano-4-oxo-3,7diphenyl-3,4-dihydrophthalazine-1-carboxylate (6), respectively. 


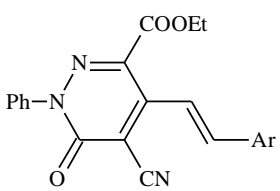

$\mathbf{5 a}, \mathbf{b}$

a, $\mathrm{Ar}=\mathrm{C}_{6} \mathrm{H}_{5} ; \mathbf{b}, \mathrm{Ar}=2-\mathrm{OH}-\mathrm{C}_{6} \mathrm{H}_{4}$

$$
\mathrm{ArCHO} \uparrow \mathrm{EtOH} / \text { pip. }
$$

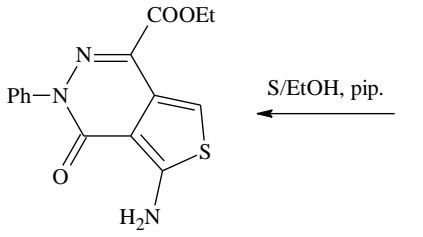

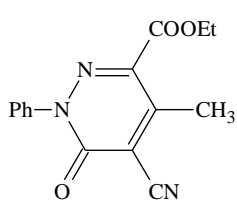

2

$\mathrm{NH}_{2} \mathrm{NH}_{2} \cdot \mathrm{H}_{2} \mathrm{O} \mid \mathrm{EtOH}$<smiles>Cc1c(C=N)nn(P)c(=O)c1C#N</smiles>

Scheme 2

The structural assignment of these compounds $\mathbf{4}, \mathbf{5 a}$ and $\mathbf{5 b}$ were supported by elemental analysis and spectral data in experimental section.

The formation of compound $\mathbf{6}$ may be proceeding by the following mechanistic equation:<smiles>CCOC(=O)c1nn(-c2ccccc2)c(=O)c(C#N)c1C(=O)ON=C(C#N)/C=C(\C#N)c1ccccc1</smiles>

Scheme 3

The structure of compound $\mathbf{6}$ was supported by elemental analysis and spectral data. The IR spectrum showed absorption bands at 3448, $3301\left(\mathrm{NH}_{2}\right), 2204$ $(\mathrm{C} \equiv \mathrm{N}), 1720(\mathrm{C}=\mathrm{O})$ and $1660(\mathrm{C}=\mathrm{O}) \mathrm{cm}^{-1}$. The ${ }^{1} \mathrm{H}$ NMR spectrum showed signals at $\delta 1.31$ and $4.39 \mathrm{ppm}$ for ethyl ester group, in addition to signal at $\delta$ $8.22 \mathrm{ppm}$ for $\mathrm{NH}_{2}$.

While,

5-cyano-4-methyl-6-oxo-1-phenyl-1,6-dihydropyridazine-3carbohydrazide (7) was obtained by reaction of $\mathbf{2}$ with hydrazine hydrate in 
refluxing ethanol. The structure of 7 was supported by elemental analysis, absence of IR band and ${ }^{1} \mathrm{H}$ NMR signal for the ester group.

Carbohydrazide $\mathbf{7}$ was refluxed with benzaldehyde derivatives namely, 4-chlorobenzaldehyde, vanillin and/or salicylaldehyde, in acetic acid to gave the hydrazone derivatives 8a-c, respectively. The structures of compounds 8a-c were established on the basis of their elemental analysis and spectral data. The IR spectrums of 8a-c showed absorption bands at $1595-1613 \mathrm{~cm}^{-1}$ for $\mathrm{C}=\mathrm{N}$ hydrazine band. The ${ }^{1} \mathrm{H}$ NMR spectrum of $8 \mathbf{a}\left(\mathrm{DMSO}-\mathrm{d}_{6}\right)$ displayed the following $\delta$ ppm values: $2.66\left(\mathrm{~s}, 3 \mathrm{H}, \mathrm{CH}_{3}\right), 7.48-8.42(\mathrm{~m}, 10 \mathrm{H}, \mathrm{Ar}-\mathrm{H}+\mathrm{N}=\mathrm{CH}), 12.05(\mathrm{~s}, 1 \mathrm{H}$, $\mathrm{NH})$.

When carbohydrazide 7 was heated under reflux with acetic anhydride and/or phthalic anhydride, it furnished the $N^{\prime}, N^{\prime}$-diacetyl-5-cyano-4-methyl-6-oxo-1phenyl-1,6-dihydropyridazine-3-carbohydrazide (9) and 5-cyano- $N$-(1,3-dioxo1,3-dihydro-2 $H$-isoindol-2-yl)-4-methyl-6-oxo-1-phenyl-1,6-dihydropyridazine-3carboxamide (10), respectively.

The structures of compounds $\mathbf{9}$ and $\mathbf{1 0}$ were established on the basis of their elemental analysis and spectral data. IR spectrum of $\mathbf{9}$ showed absorption bands at $3526(\mathrm{NH})$ and $1721(\mathrm{C}=\mathrm{O}) \mathrm{cm}^{-1}$. The ${ }^{1} \mathrm{H}$ NMR spectrum of 9 displayed signal at $\delta 2.32 \mathrm{ppm}$ characteristic for $2 \mathrm{CH}_{3} \mathrm{CO}$ in addition to signal at $\delta 11.08 \mathrm{ppm}$ for $\mathrm{NH}$. The IR spectrum of compound $\mathbf{1 0}$ showed absorption bands at 1790, 1741, 1706 and $1672 \mathrm{~cm}^{-1}$ characteristic for carbonyl groups. 


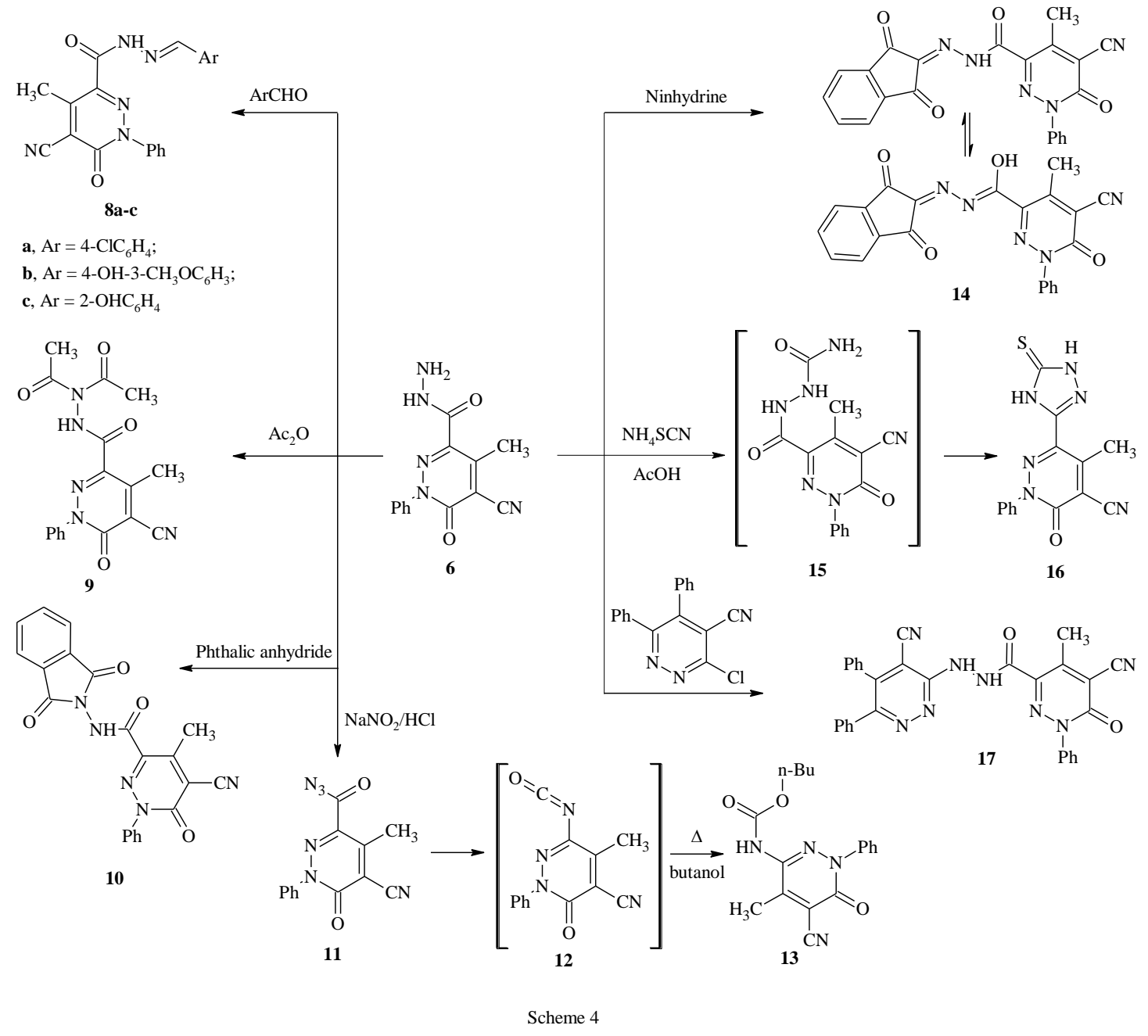

Diazotization of 7 with sodium nitrite in glacial acetic acid at $0{ }^{\circ} \mathrm{C}$ afforded 5cyano-4-methyl-6-oxo-1-phenyl-1,6-dihydropyridazine-3-carbonyl azide (11) which showed in its IR spectrum absorption band at $2186 \mathrm{~cm}^{-1}$ characteristic for azide group. When compound $\mathbf{1 1}$ was boiled in butanol afforded butyl (5-cyano4-methyl-6-oxo-1-phenyl-1,6-dihydropyridazin-3-yl)carbamate (13) without isolation of the intermediate 12. The IR spectrum of $\mathbf{1 3}$ showed absorption band at $1726 \mathrm{~cm}^{-1}$ characteristic for ester group. The ${ }^{1} \mathrm{H}$ NMR spectrum of $\mathbf{1 3}$ showed signals at $\delta 0.89,1.30,1.56$ and $4.09 \mathrm{ppm}$ characteristic for $n$-butyl ester group, in addition to signal at $\delta 9.84 \mathrm{ppm}$ for $\mathrm{NH}$.

Treatment of compound 7 with ninhydrine and/or ammonium thiocyanate in acetic acid afforded 5-cyano- $N^{\prime}$-(1,3-dioxo-1,3-dihydro- $2 \mathrm{H}$-inden-2ylidene)-4-methyl-6-oxo-1-phenyl-1,6-dihydropyridazine-3-carbohydrazide (14) and 5-methyl-3-oxo-2-phenyl-6-(5-thioxo-4,5-dihydro-1 $H$-1,2,4-triazol-3-yl)-2,3dihydropyridazine-4-carbonitrile (16), respectively. The structures of compounds 
14 and 16 were established on the basis of their elemental analysis and spectral data. The IR spectrum of $\mathbf{1 4}$ showed characteristic absorption bands at 1737 $(\mathrm{C}=\mathrm{O}), 1680(\mathrm{C}=\mathrm{O})$ and $1585(\mathrm{C}=\mathrm{C}) \mathrm{cm}^{-1} .{ }^{1} \mathrm{H}$ NMR spectrum of 14 showed signal at $\delta 13.84 \mathrm{ppm}$ characteristic for $\mathrm{NH}$. Also, the IR spectrum of $\mathbf{1 6}$ showed characteristic absorption bands at 3349, $3168(\mathrm{NH})$ and $1374(\mathrm{C}=\mathrm{S})$ $\mathrm{cm}^{-1}$. ${ }^{1} \mathrm{H}$ NMR spectrum of 16 showed signals at $\delta 9.49$ and $10.53 \mathrm{ppm}$ characteristic for $\mathrm{NH}$.

Equimolar amounts of carbohydrazide $\mathbf{7}$ and 3-chloro-4-cyano-5,6diphenylpyridazine in n-butanol were refluxed to afford 5-cyano-N'-(4-cyano5,6-diphenyl-3-pyridazinyl)-4-methyl-6-oxo-1-phenyl-1,6-dihydro-3-pyridazinecarbohydrazide (17). The structure of $\mathbf{1 7}$ was established by elemental analysis. Also, the IR spectrum exhibited characteristic absorption bands at 3434, 3059 $(\mathrm{NH}), 2230(\mathrm{C} \equiv \mathrm{N}) \mathrm{cm}^{-1}$ and the ${ }^{1} \mathrm{H}$ NMR spectrum showed characteristic signals at $\delta$ 7.23-7.69 ppm for $(\mathrm{NH}+\mathrm{Ar}-\mathrm{H})$.

\subsection{Electroanalytical behavior of some synthesized pyridazines}

The simplest behaviour was exhibited by the pyridazine derivative (2). Three successive oxidation steps were observed at $-1232,172$ and $1056 \mathrm{mV}$ (vs. SCE). While, the reverse scan show three different reduction peaks at $-495,-1438$ and $-2136 \mathrm{mV}$ (vs. SCE). Voltammetric investigation performed at a sweep rate of $0.25 \mathrm{~V} \mathrm{~s}^{-1}$ indicates reversible systems [9].

Figure 1, shows the cyclic voltammogram of ethyl 5-cyano-4-[2-(2-hydroxyphenyl)ethenyl]-6-oxo-1-phenyl-1,6-dihydropyridazine-3-carboxylate $\mathbf{5 b}$ in $\mathrm{DMF} / \mathrm{Et}_{4} \mathrm{NClO}_{4}$ from $-3 \mathrm{~V}$ to $2 \mathrm{~V}$ at a scan rate of $0.25 \mathrm{Vs}^{-1}$.

An anodic oxidation peak appears at $211 \mathrm{mV}$ presumably corresponding to formation of ethyl 4-(1-benzofuran-2-yl)-5-cyano-6-oxo-1-phenyl-1,6-dihydropyridazine-3-carboxylate [10], while the reverse scan show reduction peaks at $-544,-1349$ and $-2057 \mathrm{mV}$ (Similar to compound 2).<smiles>CCOC(=O)c1nn(-c2ccccc2)c(=O)c(C#N)c1/C=C/c1ccccc1O</smiles>

$5 b$

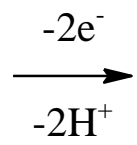<smiles>CCOC(=O)c1nn(-c2ccccc2)c(=O)c(C#N)c1-c1cc2ccccc2o1</smiles>

18 


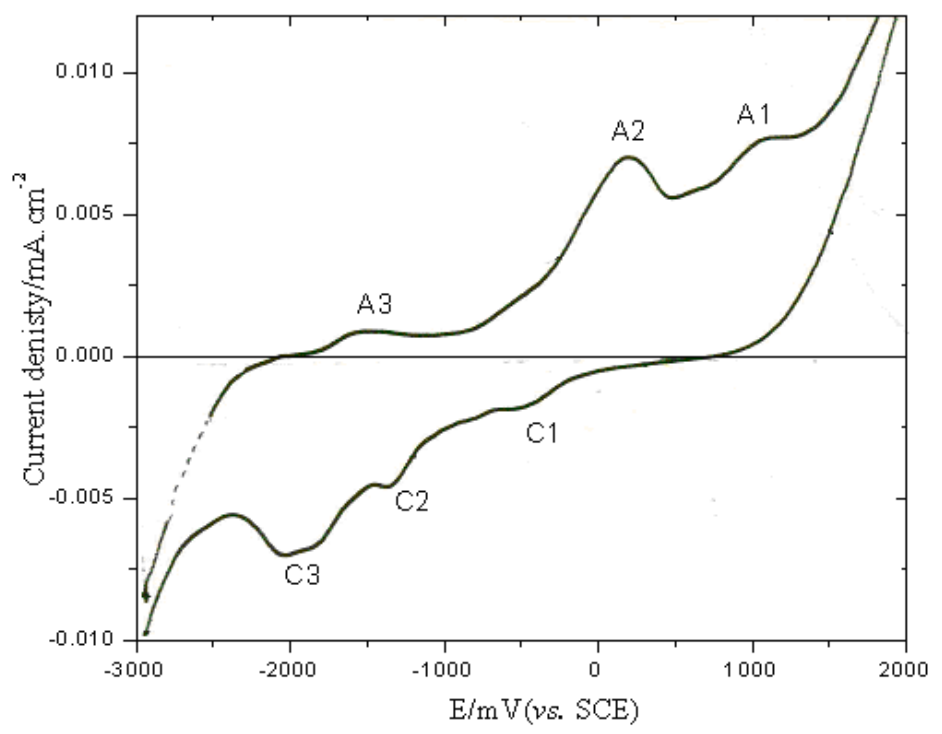

Figure 1: Cyclic voltammogram on $10^{-3}$ solution of compound $\mathbf{5 b}$ in $\mathrm{DMF} / \mathrm{Et}_{4} \mathrm{NClO}_{4}$ at a platinum electrode with scan rate $0.25 \mathrm{Vs}^{-1}$.

Clear differences appear for the carbohydrazide derivative 7 (Fig. 2). The second wave A2 corresponds to an irreversible transfer, but is located ( $398 \mathrm{mV}$ vs. SCE) with high oxidation current at more positive potential than the second oxidation peak of substrate 2 .

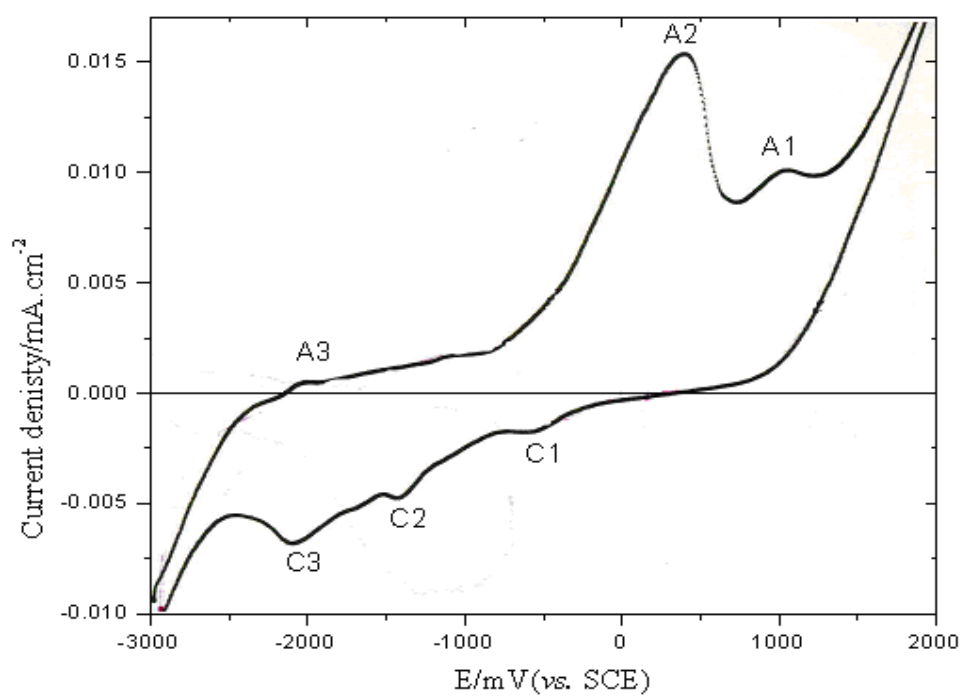

Fig. 2: Cyclic voltammogram on $10^{-3}$ solution of compound 7 in $\mathrm{DMF} / \mathrm{Et}_{4} \mathrm{NClO}_{4}$ at a platinum electrode with scan rate $0.25 \mathrm{Vs}^{-1}$. 
The mechanism suggested by Lund [11] can explain these results: the electrochemical oxidation is probably a 4-electron process leading to intermediate formation of an acylium cation.<smiles>Cc1c(C(=O)NN)nn(P)c(=O)c1C#N</smiles><smiles>Cc1c(C(=O)N=N)nn([TlH])c(=O)c1C#N</smiles>

7<smiles>Cc1c(C(=O)O)n[nH]c(=O)c1C#N</smiles>

20<smiles>Cc1c(C(=O)NNC(=O)c2nn(-c3ccccc3)c(=O)c(C)c2C)nn(P)c(=O)c1C(=O)NN</smiles>

Scheme 5

The latter could react either with water to give the parent acid 20 or with the starting compound leading to 5-cyano-N'-[(5-cyano-4-methyl-6-oxo-1-phenyl-1,6dihydro-3-pyridazinyl)carbonyl]-4-methyl-6-oxo-1-phenyl-1,6-dihydro-3-

pyridazinecarbohydrazide 19 (which is further oxidized into nitrogen and the parent acid 20). While, the reverse scan show three different reduction peaks at $-564,-1428$ and $-2098 \mathrm{mV}$ (vs. SCE). Presumably, the hydrazide was reduced to the corresponding amide 21 [12]. The amide may, after uptake of two electrons, lose an amino or a hydroxyl group, and then be further reduced to a methanol or an aminomethylpyridazine derivative, beside the compound may be reduced in the pyridazine ring [13].<smiles>Cc1c(C(=O)NN)nn(-c2ccccc2)c(=O)c1C#N</smiles>

7<smiles>C[SiH2]</smiles><smiles>Cc1c(C(N)=O)nn(-c2ccccc2)c(=O)c1C#N</smiles>

21

The carbohydrazide derivative $\mathbf{8 b}$ displays a totally different behavior. Anodic oxidation peaks of $8 \mathrm{~b}$ appears at $-1340,-73$ and $1115 \mathrm{mV}$ (vs. SCE) 
corresponding to an oxidation processes often leading to formation of oxadiazole derivative $22[14,15]$ with a relatively low oxidation current.<smiles>COc1cc(/C=N/NC(=O)c2nn(-c3ccccc3)c(=O)c(C)c2C)ccc1O</smiles>

8b<smiles>COc1cc(-c2nnc(-c3nn(-c4ccccc4)c(=O)c(C)c3C)o2)ccc1O</smiles>

22

\subsection{Screening for an antimicrobial activity}

Compounds 2, 6, 7, 8c and 10 were tested for in vitro antimicrobial activity using the method described by Heatly [15]. Tetracycline was used as antibacterial agent and amphotericn B as antifungal agent standards. The zone of inhibition of bacterial growth around the disc was observed. The screening results given in Table 1 indicate that all the compounds have antibacterial activities against Escherichia coli and Staphylococcus aureus except compound $\mathbf{6}$ but not antifungal activities on tested microorganisms.

Table 1: In vitro antimicrobial activities of some of the prepared compounds ${ }^{\mathrm{a}}$

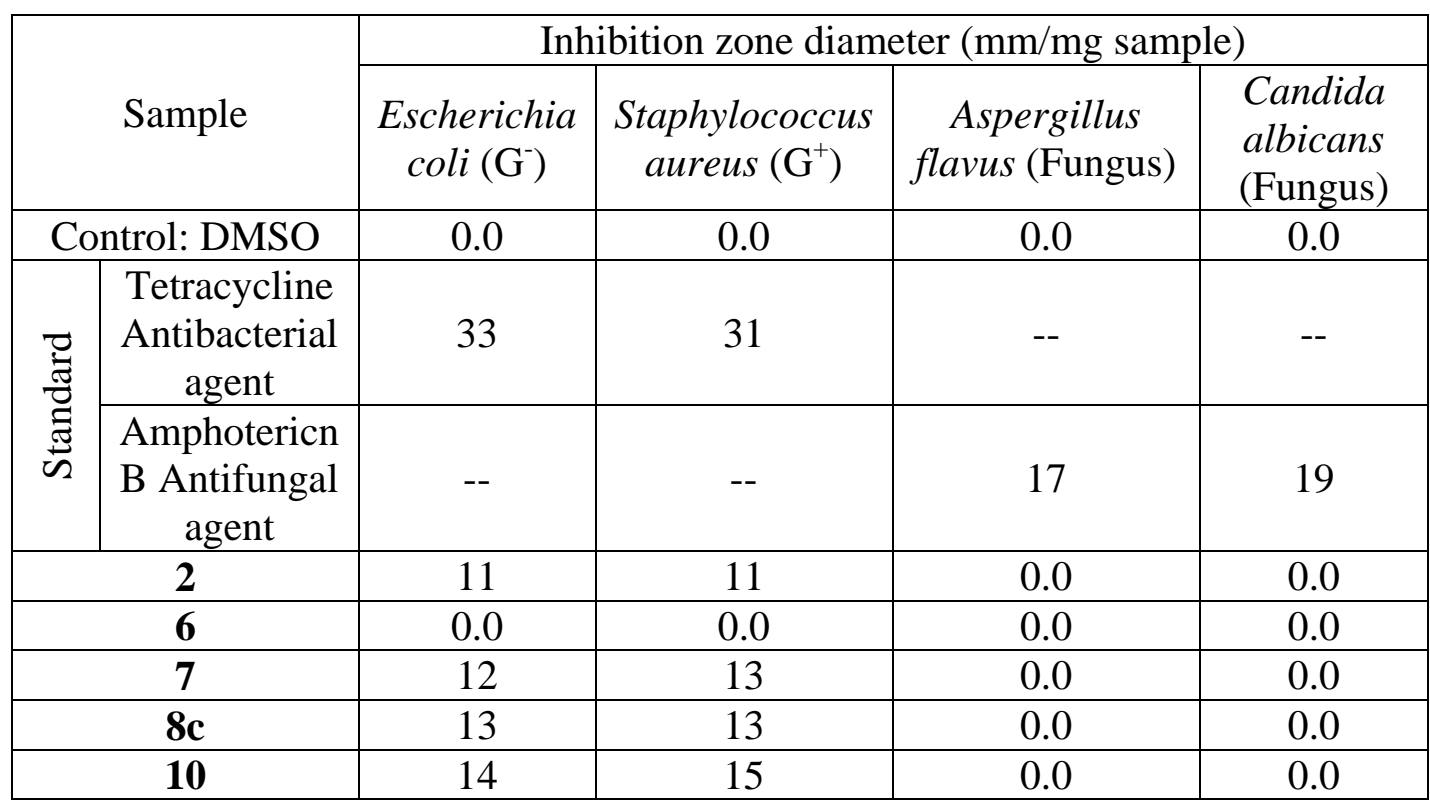




\section{Experimental}

\subsection{Instrumentation}

All melting points are uncorrected and were determined on Gallenkamp electric melting point apparatus. IR spectra ( $\mathrm{KBr}$ discs) were recorded on a FT/IR-400 spectrophotometer (Perkin Elmer). ${ }^{1} \mathrm{H}$ NMR spectra were recorded on a varian300 (DMSO-d6) solution. Chemical shifts were reported as $\delta$ values relative to tetramethylsilane (TMS) as internal reference. The analyses and in vitro antimicrobial activities were carried out at Micro Analytical Center, Cairo University.

Ethyl 3-oxo-2-(phenylhydrazono)butanoate (1a) and 1-(phenylhydrazono) propan-2-one (1b) were prepared according to the methods described by [17].

\subsection{Synthesis}

\section{Ethyl 5-cyano-4-methyl-6-oxo-1-phenyl-1,6-dihydropyridazine-3-carboxylate} (2)

A mixture of compound 1a $(0.01 \mathrm{~mol})$, ethyl cyanoacetate $(0.01 \mathrm{~mol})$ and ammonium acetate $(2 \mathrm{~g})$ in acetic acid $(30 \mathrm{ml})$ was heated under reflux for $3 \mathrm{~h}$. The solid product obtained upon cooling was filtered off, dried and recrystallized from ethanol to give $\mathbf{2}$.

Yield: 56\%; yellow crystals; m.p. 166-168 ${ }^{\circ} \mathrm{C}$. IR: 3072, 2980, $2931(\mathrm{CH}), 2230$ $(\mathrm{C} \equiv \mathrm{N}), 1717(\mathrm{C}=\mathrm{O}), 1676(\mathrm{C}=\mathrm{O})$ and $1585(\mathrm{C}=\mathrm{C}) \mathrm{cm}^{-1} .{ }^{1} \mathrm{H}$ NMR $\left(\mathrm{DMSO}-\mathrm{d}_{6}\right): \delta=$ $1.28\left(\mathrm{t}, 3 \mathrm{H}, \mathrm{CH}_{3}\right), 2.64\left(\mathrm{~s}, 3 \mathrm{H}, \mathrm{CH}_{3}\right), 4.34\left(\mathrm{q}, 2 \mathrm{H}, \mathrm{CH}_{2}\right), 7.54-7.57(\mathrm{~m}, 5 \mathrm{H}, \mathrm{Ar}-\mathrm{H})$.

Anal: $\mathrm{C}_{15} \mathrm{H}_{13} \mathrm{~N}_{3} \mathrm{O}_{3}$ (283.28); Calcd: C, 63.60; H, 4.63; N, 14.83; Found: C, 63.40; $\mathrm{H}, 4.48 ; \mathrm{N}, 14.92$.

\section{6-acetyl-3-imino-2,5-diphenyl-2,3,4,5-tetrahydropyridazine-4-carbonitrile (3)}

A mixture of compound $\mathbf{1 b}(0.01 \mathrm{~mol})$ and benzylidene malononitrile $(0.01 \mathrm{~mol})$ in pyridine $(30 \mathrm{ml})$ was heated under reflux for $3 \mathrm{~h}$. The reaction mixture was cooled, poured into cold water and neutralized with dil. $\mathrm{HCl}$. The separated solid product was filtered off, dried and recrystallized from ethanol to give 3 .

Yield: 62\%; yellow crystals; m.p. 230-232 ${ }^{\circ} \mathrm{C}$. IR: 3407 (NH), 3313, 3207, 3066 $(\mathrm{CH}), 2188(\mathrm{C} \equiv \mathrm{N}), 1644(\mathrm{C}=\mathrm{O})$ and $1593(\mathrm{C}=\mathrm{C}) \mathrm{cm}^{-1} .{ }^{1} \mathrm{H}$ NMR $\left(\mathrm{DMSO}-d_{6}\right): \delta=$ 2.32(s, 3H, $\left.\mathrm{CH}_{3}\right), 4.75,5.99(\mathrm{~s}, 2 \mathrm{H}, 2 \mathrm{CH}), 7.17(\mathrm{~s}, 1 \mathrm{H}, \mathrm{NH}), 7.20-7.53(\mathrm{~m}, 10 \mathrm{H}, \mathrm{Ar}-$ $\mathrm{H})$.

Anal: $\mathrm{C}_{19} \mathrm{H}_{16} \mathrm{~N}_{4} \mathrm{O}$ (316.36); Calcd: C, 72.13; H, 5.10; N, 17.71; Found: C, 72.25; $\mathrm{H}, 4.93 ; \mathrm{N}, 17.50$. 


\section{Ethyl 5-amino-4-oxo-3-phenyl-3,4-dihydrothieno[3,4- $d$ ]pyridazine-1- carboxylate (4)}

A mixture of compound $2(0.005 \mathrm{~mol})$, sulphur $(0.005 \mathrm{~mol})$ and catalytic amount of piperidine in absolute ethanol $(60 \mathrm{ml})$ was heated under reflux for $4 \mathrm{~h}$. The solid product obtained upon cooling was filtered off, dried and recrystallized from $n$ butanol to give 4.

Yield: 59\%; orange crystals; m.p. 180-182 ${ }^{\circ} \mathrm{C}$. IR: 3419, $3302\left(\mathrm{NH}_{2}\right), 1707(\mathrm{C}=\mathrm{O})$, $1642(\mathrm{C}=\mathrm{O})$ and $1588(\mathrm{C}=\mathrm{C}) \mathrm{cm}^{-1} .{ }^{1} \mathrm{H}$ NMR (DMSO- $\left.d_{6}\right): \delta=1.27\left(\mathrm{t}, 3 \mathrm{H}, \mathrm{CH}_{3}\right)$, 4.33(q, 2H, $\left.\mathrm{CH}_{2}\right), 7.08\left(\mathrm{~s}, 2 \mathrm{H}, \mathrm{NH}_{2}\right), 7.10-7.61(\mathrm{~m}, 6 \mathrm{H}$. Ar-H).

Anal: $\mathrm{C}_{15} \mathrm{H}_{13} \mathrm{~N}_{3} \mathrm{O}_{3} \mathrm{~S}$ (315.35); Calcd: C, 57.13; H, 4.16; N, 13.33; Found: C, 57.21; H, 4.28; N, 13.15 .

Ethyl 5-cyano-6-oxo-1-phenyl-4-[2-phenylethenyl]-1,6-dihydro-pyridazine-3carboxylate (5a) and ethyl 5-cyano-4-[2-(2-hydroxy-phenyl)ethenyl]-6-oxo-1phenyl-1,6-dihydropyridazine-3-carboxylate $(5 \mathrm{~b})$

A mixture of compound 2 (0.005 mol), benzaldehyde and/or salicylaldehyde $(0.005 \mathrm{~mol})$ in presence of catalytic amount of piperidine in absolute ethanol (60 $\mathrm{ml}$ ) was heated under reflux for $4 \mathrm{~h}$. The solid product obtained upon cooling was filtered off, dried and recrystallized from the proper solvent to give $\mathbf{5 a}$ and $\mathbf{5 b}$, respectively.

Compound 5a

Yield: 76\%; from $n$-butanol as Yellow crystals; m.p. $218-220{ }^{\circ} \mathrm{C}$. IR: 3429,3064 $(\mathrm{CH}), 2227(\mathrm{C} \equiv \mathrm{N}), 1723(\mathrm{C}=\mathrm{O}), 1671(\mathrm{C}=\mathrm{O})$ and $1618(\mathrm{C}=\mathrm{C}) \mathrm{cm}^{-1} \cdot{ }^{1} \mathrm{H}$ NMR $\left(\mathrm{DMSO}-d_{6}\right): \delta=1.28\left(\mathrm{t}, 3 \mathrm{H}, \mathrm{CH}_{3}\right), 4.37\left(\mathrm{q}, 2 \mathrm{H}, \mathrm{CH}_{2}\right), 7.49-7.74(\mathrm{~m}, 12 \mathrm{H}, \mathrm{Ar}-\mathrm{H}$ and olefinic protons).

Anal: $\mathrm{C}_{22} \mathrm{H}_{17} \mathrm{~N}_{3} \mathrm{O}_{3}$ (371.39); Calcd: C, 71.15; H, 4.61; N, 11.31; Found: C, 71.26; $\mathrm{H}, 4.45 ; \mathrm{N}, 11.19$.

Compound 5b

Yield: 31\%; from ethanol as violet crystals; m.p. $256-258{ }^{\circ} \mathrm{C}$. IR: $3352(\mathrm{OH})$, 3069, $2973(\mathrm{CH}), 2227(\mathrm{C} \equiv \mathrm{N}), 1734(\mathrm{C}=\mathrm{O}), 1654(\mathrm{C}=\mathrm{O})$ and $1608(\mathrm{C}=\mathrm{C}) \mathrm{cm}^{-1}$.

Anal: $\mathrm{C}_{22} \mathrm{H}_{17} \mathrm{~N}_{3} \mathrm{O}_{4}$ (387.39); Calcd: C, 68.21; H, 4.42; N, 10.85; Found: C, 68.12; $\mathrm{H}, 4.31 ; \mathrm{N}, 10.67$.

\section{Ethyl 5-amino-6-cyano-4-oxo-3,7-diphenyl-3,4-dihydrophthalazine-1- carboxylate (6)}

A mixture of compound $2(0.005 \mathrm{~mol})$, benzylidene malononitrile $(0.005 \mathrm{~mol})$ and catalytic amount of piperidine in absolute ethanol $(60 \mathrm{ml})$ was heated under reflux for $3 \mathrm{~h}$. The solid product separated during heating was filtered off, dried and recrystallized from DMF to give $\mathbf{6}$. 
Yield: 34\%; yellow crystals; m.p. 284-286 ${ }^{\circ} \mathrm{C}$. IR: 3448, $3301\left(\mathrm{NH}_{2}\right), 3117,3061$, 2985, $2933(\mathrm{CH}), 2204(\mathrm{C} \equiv \mathrm{N}), 1720(\mathrm{C}=\mathrm{O}), 1660(\mathrm{C}=\mathrm{O})$ and $1598(\mathrm{C}=\mathrm{C}) \mathrm{cm}^{-1}$. ${ }^{1} \mathrm{H}$ NMR (DMSO- $\left.d_{6}\right): \delta=1.31\left(\mathrm{t}, 3 \mathrm{H}, \mathrm{CH}_{3}\right), 4.39\left(\mathrm{q}, 2 \mathrm{H}, \mathrm{CH}_{2}\right), 7.55-8.22(\mathrm{~m}, 11 \mathrm{H}$, Ar- $\mathrm{H}$ and $\mathrm{NH}_{2}$ ).

Anal: $\mathrm{C}_{24} \mathrm{H}_{18} \mathrm{~N}_{4} \mathrm{O}_{3}$ (410.42); Calcd: C, 70.23; H, 4.42; N, 13.65; Found: C, 70.12; $\mathrm{H}, 4.50 ; \mathrm{N}, 13.45$.

\section{5-cyano-4-methyl-6-oxo-1-phenyl-1,6-dihydropyridazine-3-carbohydrazide} (7)

A mixture of compound $2(0.005 \mathrm{~mol})$ and hydrazine hydrate $(0.01 \mathrm{~mol})$ in ethanol $(60 \mathrm{ml})$ was heated under reflux on a water bath for $5 \mathrm{~h}$. The solid product obtained upon cooling was filtered off, dried and recrystallized from $n$-butanol to give 7.

Yield: 84\%; brown crystals; m.p. 236-238 ${ }^{\circ} \mathrm{C}$. IR: 3437-3331 (NH and $\left.\mathrm{NH}_{2}\right), 2928$ $(\mathrm{CH}), 2242(\mathrm{C} \equiv \mathrm{N})$ and $1680(\mathrm{C}=\mathrm{O}) \mathrm{cm}^{-1} .{ }^{1} \mathrm{H}$ NMR $\left(\mathrm{DMSO}-d_{6}\right): \delta=2.57(\mathrm{~s}, 3 \mathrm{H}$, $\left.\mathrm{CH}_{3}\right), 4.60$ (s, 2H, NH 2 ), 7.48-7.70 (m, 5H, Ar-H), 9.88 (s, broaded, 1H, NH).

Anal: $\mathrm{C}_{13} \mathrm{H}_{11} \mathrm{~N}_{5} \mathrm{O}_{2}$ (269.26); Calcd: C, 57.99; H, 4.12; N, 26.01; Found: C, 58.10; $\mathrm{H}, 4.28 ; \mathrm{N}, 25.92$.

$N^{\prime}$-[(4-chlorophenyl)methylidene]-5-cyano-4-methyl-6-oxo-1-phenyl-1,6dihydropyridazine-3-carbohydrazide (8a), 5-cyano- $N$ '-[(4-hydroxy-3-methoxyphenyl)methylidene]-4-methyl-6-oxo-1-phenyl-1,6-dihydropyridazine-3carbohydrazide (8b) and 5-cyano- $N^{\prime}$-[(2-hydroxyphenyl)methylidene]-4methyl-6-oxo-1-phenyl-1,6-dihydropyridazine-3-carbohydrazide (8c)

A mixture of compound $7(0.005 \mathrm{~mol})$ and benzaldehyde derivatives namely, [4chlorobenzaldehyde, vanillin and/or salicylaldehyde], $(0.005 \mathrm{~mol})$ in acetic acid $(30 \mathrm{ml})$ was refluxed for $3 \mathrm{~h}$. The solid product obtained upon cooling was filtered off, dried and recrystallized from the proper solvent to give 8a-c, respectively.

\section{Compound 8a}

Yield: $46 \%$; from $n$-butanol as green crystals; m.p. 246-248 ${ }^{\circ} \mathrm{C}$. IR: 3433 (NH), 3298, $2927(\mathrm{CH}), 2236(\mathrm{C} \equiv \mathrm{N})$ and $1685(\mathrm{C}=\mathrm{O}) \mathrm{cm}^{-1} .{ }^{1} \mathrm{H}$ NMR (DMSO-d $\left.d_{6}\right): \delta=$ $2.66\left(\mathrm{~s}, 3 \mathrm{H}, \mathrm{CH}_{3}\right), 7.48-8.42(\mathrm{~m}, 10 \mathrm{H}, \mathrm{Ar}-\mathrm{H}+\mathrm{N}=\mathrm{CH}), 12.05$ (s, 1H, NH).

Anal: $\mathrm{C}_{20} \mathrm{H}_{14} \mathrm{ClN}_{5} \mathrm{O}_{2}$ (391.81); Calcd: C, 61.31; H, 3.60; N, 17.87; Found: C, $61.44 ; \mathrm{H}, 3.46 ; \mathrm{N}, 17.93$.

\section{Compound 8b}

Yield: 84\%; from acetic acid as yellow crystals; m.p. 258-260 ${ }^{\circ} \mathrm{C}$. IR: $3421(\mathrm{OH})$, 3072, $2971(\mathrm{CH}), 2230(\mathrm{C} \equiv \mathrm{N}), 1677(\mathrm{C}=\mathrm{O})$ and $1595(\mathrm{C}=\mathrm{N}) \mathrm{cm}^{-1}$.

Anal: $\mathrm{C}_{21} \mathrm{H}_{17} \mathrm{~N}_{5} \mathrm{O}_{4}$ (403.39); Calcd: C, 62.53; H, 4.25; N, 17.36; Found: C, 62.44; $\mathrm{H}, 4.15 ; \mathrm{N}, 17.45$. 


\section{Compound 8c}

Yield: 86\%; from acetic acid as grey crystals; m.p. $268-270{ }^{\circ} \mathrm{C}$. IR: $3413(\mathrm{OH})$, 3065, $2977(\mathrm{CH}), 2232(\mathrm{C} \equiv \mathrm{N}), 1680(\mathrm{C}=\mathrm{O})$ and $1613(\mathrm{C}=\mathrm{N}) \mathrm{cm}^{-1}$.

Anal: $\mathrm{C}_{20} \mathrm{H}_{15} \mathrm{~N}_{5} \mathrm{O}_{3}$ (373.36); Calcd: C, 64.34; H, 4.05; N, 18.76; Found: C, 64.20; $\mathrm{H}, 3.94 ; \mathrm{N}, 18.91$.

\section{$N^{\prime}, N^{\prime}$-diacetyl-5-cyano-4-methyl-6-oxo-1-phenyl-1,6-dihydro-pyridazine-3- carbohydrazide (9)}

A mixture of compound $7(0.005 \mathrm{~mol})$ and acetic anhydride $(5 \mathrm{ml})$ was heated under reflux for $1 \mathrm{~h}$. The acetic anhydride excess was evaporated under reduced pressure and the solid product obtained was recrystallized from ethanol to give $\mathbf{9}$.

Yield: 10\%; pale brown crystals; m.p. 194-196 ${ }^{\circ} \mathrm{C}$. IR: 3577, $3526(\mathrm{NH}), 3255$, 3071, 3000, $2935(\mathrm{CH}), 2240(\mathrm{C} \equiv \mathrm{N}), 1721(\mathrm{C}=\mathrm{O}), 1689(\mathrm{C}=\mathrm{O})$ and $1592(\mathrm{C}=\mathrm{C})$ $\mathrm{cm}^{-1} .{ }^{1} \mathrm{H}$ NMR (DMSO- $\left.d_{6}\right): \delta=2.32\left(\mathrm{~s}, 6 \mathrm{H}, 2 \mathrm{CH}_{3} \mathrm{CO}\right), 2.63\left(\mathrm{~s}, 3 \mathrm{H}, \mathrm{CH}_{3}\right), 7.51-$ 7.71(m, 5H, Ar-H), 11.08(s, 1H, NH).

Anal: $\mathrm{C}_{17} \mathrm{H}_{15} \mathrm{~N}_{5} \mathrm{O}_{4}$ (353.33); Calcd: C, 57.79; H, 4.28; N, 19.82; Found: C, 57.62; $\mathrm{H}, 4.41 ; \mathrm{N}, 19.91$.

\section{5-cyano- $N$-(1,3-dioxo-1,3-dihydro-2H-isoindol-2-yl)-4-methyl-6-oxo-1-phenyl- 1,6-dihydropyridazine-3-carboxamide (10)}

A mixture of compound $7(0.005 \mathrm{~mol})$ and phthalic anhydride $(0.005 \mathrm{~mol})$ in acetic acid $(30 \mathrm{ml})$ was refluxed for $3 \mathrm{~h}$. The solid product obtained upon cooling was filtered off, dried and recrystallized from acetic acid to give $\mathbf{1 0 .}$

Yield: 56\%; white crystals; m.p. 264-266 ${ }^{\circ} \mathrm{C}$. IR: 3439, $3331(\mathrm{NH}), 3100,2933$ $(\mathrm{CH}), 2240(\mathrm{C} \equiv \mathrm{N}), 1790-1741$ (imidic $\mathrm{C}=\mathrm{O}$ ) and 1672 (cyclic and acyclic amide $\mathrm{C}=\mathrm{O}) \mathrm{cm}^{-1} .{ }^{1} \mathrm{H}$ NMR (DMSO- $\left.d_{6}\right): \delta=2.64\left(\mathrm{~s}, 3 \mathrm{H}, \mathrm{CH}_{3}\right), 7.56-8.01(\mathrm{~m}, 9 \mathrm{H}, \mathrm{Ar}-\mathrm{H})$, $11.70(\mathrm{~s}, 1 \mathrm{H}, \mathrm{NH})$.

Anal: $\mathrm{C}_{21} \mathrm{H}_{13} \mathrm{~N}_{5} \mathrm{O}_{4}$ (399.36); Calcd: C, 63.16; H, 3.28; N, 17.54; Found: C, 63.32; $\mathrm{H}, 3.11 ; \mathrm{N}, 17.69$.

5-cyano-4-methyl-6-oxo-1-phenyl-1,6-dihydropyridazine-3-carbonyl azide (11)

A stirred cold solution of compound $7(0.005 \mathrm{~mol})$ in acetic acid $(20 \mathrm{ml})$ was treated dropwise with a cold solution of $\mathrm{NaNO}_{2}(0.01 \mathrm{~mol})$ in water $(5 \mathrm{ml})$. The reaction mixture was further stirred for $30 \mathrm{~min}$. and the separated solid product was filtered off, washed with water, dried to give 11, sufficiently pure for direct use in the next stage.

Yield: 36\%; yellow crystals; m.p. 109-110 ${ }^{\circ} \mathrm{C}$. IR: 3431, $2240(\mathrm{C} \equiv \mathrm{N}), 2186\left(\mathrm{~N}_{3}\right)$, $1686(\mathrm{C}=\mathrm{O})$ and $1632(\mathrm{C}=\mathrm{N}) \mathrm{cm}^{-1}$.

Anal: $\mathrm{C}_{13} \mathrm{H}_{8} \mathrm{~N}_{6} \mathrm{O}_{2}$ (280.24); Calcd: C, 55.72; H, 2.88; N, 29.99; Found: C, 55.91; H, 2.69; N, 30.10. 
Butyl yl)carbamate (13)

(5-cyano-4-methyl-6-oxo-1-phenyl-1,6-dihydropyridazin-3-

A solution of compound $11(0.005 \mathrm{~mol})$ in $n$-butanol $(15 \mathrm{ml})$ is heated under reflux for $3 \mathrm{~h}$. The solid product obtained upon cooling was filtered off, dried and recrystallized from $n$-butanol to give $\mathbf{1 3}$.

Yield: 6\%; white crystals; m.p. 170-172 ${ }^{\circ} \mathrm{C}$. IR: $3226(\mathrm{NH}), 2966(\mathrm{CH}), 2232$ $(\mathrm{C} \equiv \mathrm{N}), 1726(\mathrm{C}=\mathrm{O}$ of carbamate ester $), 1640(\mathrm{C}=\mathrm{O})$ and $1590(\mathrm{C}=\mathrm{C}) \mathrm{cm}^{-1} \cdot{ }^{1} \mathrm{H}$ NMR (DMSO- $\left.d_{6}\right): \delta=0.89\left(\mathrm{t}, 3 \mathrm{H}, \mathrm{CH}_{3}\right), 1.30-1.38\left(\mathrm{~m}, 2 \mathrm{H}, \mathrm{CH}_{2}\right), 1.56-1.61(\mathrm{~m}, 2 \mathrm{H}$, $\left.\mathrm{CH}_{2}\right), 2.40\left(\mathrm{~s}, 3 \mathrm{H}, \mathrm{CH}_{3}\right), 4.09\left(\mathrm{t}, 2 \mathrm{H}, \mathrm{OCH}_{2}\right), 7.46-7.58(\mathrm{~m}, 5 \mathrm{H}, \mathrm{Ar}-\mathrm{H}), 9.84(\mathrm{~s}, 1 \mathrm{H}$, $\mathrm{NH})$.

Anal: $\mathrm{C}_{17} \mathrm{H}_{18} \mathrm{~N}_{4} \mathrm{O}_{3}$ (326.35); Calcd: C, 62.57; H, 5.56; N, 17.17; Found: C, 62.71; $\mathrm{H}, 5.45 ; \mathrm{N}, 17.30$.

5-cyano- $N^{\prime}$-(1,3-dioxo-1,3-dihydro-2H-inden-2-ylidene)-4-methyl-6-oxo-1phenyl-1,6-dihydropyridazine-3-carbohydrazide (14)

A mixture of compound $7(0.005 \mathrm{~mol})$ and ninhydrine $(0.005 \mathrm{~mol})$ in acetic acid $(20 \mathrm{ml})$ was heated under reflux for $1 \mathrm{~h}$. The solid product obtained during heating was filtered off, dried and recrystallized from $\mathrm{DMF} / \mathrm{EtOH}$ to give 14.

Yield: 83\%; green crystals; m.p. 252-254 ${ }^{\circ} \mathrm{C}$. IR: 3432, $3214(\mathrm{NH}), 3064,2927$ $(\mathrm{CH}), 2239(\mathrm{C} \equiv \mathrm{N}), 1737(\mathrm{C}=\mathrm{O}), 1680(\mathrm{C}=\mathrm{O})$ and $1585(\mathrm{C}=\mathrm{C}) \mathrm{cm}^{-1} .{ }^{1} \mathrm{H}$ NMR (DMSO- $\left.d_{6}\right): \delta=2.80\left(\mathrm{~s}, 3 \mathrm{H}, \mathrm{CH}_{3}\right), 7.59-8.02(\mathrm{~m}, 9 \mathrm{H}, \mathrm{Ar}-\mathrm{H}), 13.84(\mathrm{~s}, 1 \mathrm{H}, \mathrm{NH})$.

Anal: $\mathrm{C}_{22} \mathrm{H}_{13} \mathrm{~N}_{5} \mathrm{O}_{4}$ (411.37); Calcd: C, 64.23; H, 3.19; N, 17.02; Found: C, 64.10; $\mathrm{H}, 3.32 ; \mathrm{N}, 17.11$.

\section{5-methyl-3-oxo-2-phenyl-6-(5-thioxo-4,5-dihydro-1H-1,2,4-triazol-3-yl)-2,3- dihydropyridazine-4-carbonitrile (16)}

A mixture of compound $7(0.005 \mathrm{~mol})$ and ammonium thiocyanate $(0.005 \mathrm{~mol})$ in acetic acid $(20 \mathrm{ml})$ was heated under reflux for $3 \mathrm{~h}$. The solid product separated during heating was filtered off, dried and recrystallized from DMF/EtOH to give 16.

Yield: 35\%; green crystals; m.p. $258-259{ }^{\circ} \mathrm{C} . \mathrm{IR}: 3349,3168(\mathrm{NH}), 3119(\mathrm{CH})$, $2232(\mathrm{C} \equiv \mathrm{N}), 1681(\mathrm{C}=\mathrm{O}), 1632(\mathrm{C}=\mathrm{N})$ and $1374(\mathrm{C}=\mathrm{S}) \mathrm{cm}^{-1} .{ }^{1} \mathrm{H}$ NMR (DMSO$\left.d_{6}\right): \delta=2.63\left(\mathrm{~s}, 3 \mathrm{H}, \mathrm{CH}_{3}\right), 7.50-7.74(\mathrm{~m}, 5 \mathrm{H}, \mathrm{Ar}-\mathrm{H}), 9.49-10.53(\mathrm{~s}$, broaded, $2 \mathrm{H}$, $2 \mathrm{NH})$.

Anal: $\mathrm{C}_{14} \mathrm{H}_{10} \mathrm{~N}_{6} \mathrm{OS}$ (310.33); Calcd: C, 54.18; H, 3.25; N, 27.08; Found: C, 54.30; $\mathrm{H}, 3.14$; N, 26.94.

5-cyano- $N$ '-(4-cyano-5,6-diphenyl-3-pyridazinyl)-4-methyl-6-oxo-1-phenyl1,6-dihydro-3-pyridazinecarbohydrazide (17)

A mixture of compound 7 (0.005 mol) and 3-chloro-4-cyano-5,6diphenylpyridazine $(0.005 \mathrm{~mol})$ in $n$-butanol $(30 \mathrm{ml})$ was heated under reflux for 
8h. The solid product separated during heating was filtered off, dried and recrystallized from DMF to give 17.

Yield: 50\%; yellow crystals; m.p. 288-290 ${ }^{\circ} \mathrm{C}$. IR: 3434, $3059(\mathrm{NH}), 2927(\mathrm{CH})$, $2230(\mathrm{C} \equiv \mathrm{N}), 1681(\mathrm{C}=\mathrm{O})$ and $1591(\mathrm{C}=\mathrm{C}) \mathrm{cm}^{-1} .{ }^{1} \mathrm{H}$ NMR $\left(\mathrm{DMSO}-d_{6}\right): \delta=2.71(\mathrm{~s}$, $\left.3 \mathrm{H}, \mathrm{CH}_{3}\right), 7.23-7.69(\mathrm{~m}, 17 \mathrm{H}, 2 \times \mathrm{NH}+\mathrm{Ar}-\mathrm{H})$.

Anal: $\mathrm{C}_{30} \mathrm{H}_{20} \mathrm{~N}_{8} \mathrm{O}_{2}$ (524.53); Calcd: C, 68.69; H, 3.84; N, 21.36; Found: C, 68.81; $\mathrm{H}, 3.68$; N, 21.47.

\section{Electrochemistry}

Operating conditions used for analytical and small scale preparative electrochemical studies, carried out at a platinum electrode, have been detailed in a preceding paper [17]. Flow-cell electrolysis has been carried out by using a one compartment three electrode electrochemical cell. All CV experiments are carried with stagnant solutions out at a room temperature $\left(25 \pm 1{ }^{\circ} \mathrm{C}\right)$. The working and the auxiliary platinum electrodes surfaces should be renewed prior to each run. The accessible potential range depends upon the solvent and the supporting electrolyte that are used. Typical cyclic voltammogram (anodic oxidation followed by cathodic reduction) was performed [18-21].

\section{References}

[1] Coates, W. J. (1996). Pyridazines and Their Benzo Derivatives. Comprehensive Heterocyclic Chemistry II, Vol. 6, Pergamon Press, Oxford, p. 1.

[2] Vieira, L. M. C.; Fonseca, A. M.; Raposo, M. M. M.; Kirsch, G. (2004). Electrochemical and Spectroscopic Studies of Pyridazine Derivatives. Portugaliae Electrochimica Acta, 22, 11.

[3] Brooker, S.; Davidson, T. C.; Hay, S. J.; Kelly, R. J.; Kennepohl, D. K.; Plieger, P. G.; Moubaraki, B.; Murray, K. S.; Bill, E.; Bothe, E. (2001). A convenient one-pot synthesis of 3-Aamino-2,5-dihydropyridazine and pyrimidine derivatives in the presence of high surface area $\mathrm{MgO}$ as a high effective heterogeneous base catalyst. Coord. Chem Rev, 3, 216.

[4] Cheng, Y.; Ma, B.; Wudl, F. (1999). An expedient and new synthesis of pyrrolo[1,2-b]pyridazine derivatives. J Mat Chem, 9, 2183.

[5] Sayed, G. H.; El-Mobayed, M.; El-Shekeil, A. G.; Abd Elghani, E., (1990). A new route for the synthesis of tetrahydro and dihydro-3H-pyrazolo[3,4c]pyridazino[4,3-e]pyridazine ring systems. Indian Journal of Chemistry 29B, 72.

[6] Sayed, G. H.; El-Mobayed, M.; El-Shekeil, A. G.; Abd Elghani, E., (1991). Reactions of Some New 4-Pyrazolinonyl Pyridazinones. Egypt J Chem 34(1), 73. 
[7] Abd Elghani, E. (1991). Synthesis and Reactions of Some New Pyridazinones. Bull Chem Soc Jpn 64(6), 2032.

[8] Abd Elghani, E.; Assy, M. G.; Moustafa, H. Y. (1995). Electrochemical reduction of substituted pyridazines: a new access to activated pyrroles. Monatshefte für Chemie 126, 1265.

[9] Manh, G. T.; Hazard, R.; Tallec, A.; Pradere, J. P.; Dubreuil, D.; Thiam, M.; Toupet, L. (2002). Electrochemical reduction of substituted pyridazines: a new access to activated pyrroles. Electrochimica Acta, 47, 2833.

[10] Gates, B. D.; Dalidowicz, P.; Tebben, A.; Wang, S.; Swenton, J. S. (1992). J Org Chem, 57, 2135.

[11] Lund, H. (1963). Acta Chem Scand, 17, 1077.

[12] Hazard, R.; LE Floch, M.; Tallec, A. (1996). Controlled potential oxidation of some arylhydrazides. Elecwochimics Acta 41(18), 2871.

[13] Largeron, M.; Auzeil, N.; Bacque, E.; Fleury M. B. (1997). Influence of steric crowding on the electrochemical reduction of substituted tertiary pyridylcarboxamides in aqueous acidic medium. J Chem Soc Perkin Trans 2, 495.

[14] Hammerich, O.; Parker, V. D. (1972). Anodic oxidation of organic nitrogen compounds. Part I. Cyclization of 1-arylmethylenesemicarbazides. J Chem Soc Perkin Trans 1, 1718.

[15] Chiba, T.; Okimoto, M. (1992). Electrooxidative cyclization of Nacylhydrazones of aldehydes and ketones to 1,3,4-oxadiazolines and 1,3,4oxadiazoles. J Org Chem, 57, 1375.

[16] Capparelli, M. P.; Deschepper, R. E.; Swenton, J. S. (1987). Structural and solvent/electrolyte effects on the selectivity and efficiency of the anodic oxidation of para-substituted aromatic ethers. An efficient route to quinol ether ketals and quinol ethers. J Org Chem, 52, 4953.

[17] Jellinek, H. H. G.; Urwin, J. R. (1954). Polarography of Picolinic and Isonicotinic Acid and Their Amides. J Phys Chem, 58, 168.

[18] Reischl, G.; El-Mobayed, M.; Beisswenger, R.; Regier, K.; Mossmer, C. M.; Rieker, A. (1998). Oxidation-Induced Acyl Group Transfer from Hydroquinone Esters to Nucleophiles. Zeitschrift Fur Naturforschung, 53b, 765.

[19] Hutinec, A.; Ziogas, A.; El-Mobayed, M.; Rieker, A. (1998). Spirolactones of tyrosine: synthesis and reaction with nucleophiles. J Chem Soc Perkin Trans 1, 2201.

[20] El-Mobayed, M.; Ismail, N.; Abo-El-Enein, G.; Abdel-Haleem, E. (1986). Anodic Oxidation of $\alpha$-naphthol Derivative: A Facile Route to Ring Closure Products. J Chem Soc Pak, 8(3), 305.

[21] Dreher, E. L.; Brancht, J.; El-Mobayed, M.; Winter, W.; Rieker, A. (1982). Electrochemical Oxidation, VII. Syntheses and Structure of 7-tert-Butyl-2methylbenzoxazole. Chem Ber, 115, 288. 
[22] Abd Elghani, E.; Abdel-Aal, A.; Shehab, W.; EL-Mobayed, M. (2003). A Novel Method for the Synthesis of Oxazolocoumarin Derivatives. Synthesis. 9, 1373. 